\title{
A Case Report of Nasal Adenoid Cystic Carcinoma
}

\author{
Rahim $\mathrm{H}^{1 *}$, Bencheikh $\mathrm{R}^{2}$, Gliti MA ${ }^{1}$, Harmouch $\mathrm{A}^{3}$, Benbouzid $\mathrm{MA}^{2}$, Essakali $\mathrm{L}^{2}$ \\ ${ }^{1}$ Resident physician in otorhinolaryngology, Department of Otorhinolaryngology, Head and Neck Surgery, Ibn Sina University Hospital, Faculty of \\ Medicine, Mohammed V University, Rabat, Morocco \\ ${ }^{2}$ Professor of otorhinolaryngology, Department of Otorhinolaryngology, Head and Neck Surgery, Ibn Sina University Hospital, Faculty of Medicine, \\ Mohammed V University, Rabat, Morocco \\ ${ }^{3}$ Professor of Anatomopathology, Ibn Sina University Hospital, Faculty of Medicine, Mohammed V University, Rabat, Morocco
}

$\begin{array}{ll}\text { DOI: } 10.36348 / \text { sjmps.2020.v06i11.007 } & \text { | Received: 22.08.2020 | Accepted: 29.08.2020 | Published: } 28.11 .2020 \\ \text { *Corresponding author: Rahim Hanaa } & \end{array}$

Abstract

The adenoid cystic carcinoma is a rare tumor in the region of the head and neck, it's the common malignant tumor of the salivary glands. Its location in the nasal cavity is exceptional. We report in our study a case of adenoid cystic carcinoma of the nasal cavity of a 68-year-old patient who presents nasal symptoms. The CT- scan shows a tissue process in the right nasal cavity, the endoscopy showed a process in the right nasal cavity extending to the lower cornet. The histology confirmed the adenoid cystic carcinoma. The surgical treatment consisted on the excision of the entire tumor followed by radiation therapy.

Keywords: Adenoid cystic carcinoma - nasal cavity.

Copyright (C) 2020 The Author(s): This is an open-access article distributed under the terms of the Creative Commons Attribution 4.0 International License (CC BY-NC 4.0) which permits unrestricted use, distribution, and reproduction in any medium for non-commercial use provided the original author and source are credited.

\section{INTRODUCTION}

Adenoid cystic carcinoma (ACC) accounts for 3 to $5 \%$ of all head and neck malignancies [1]. It is common in females compared to males with a ratio of 2:1 and the peak incidence at age of 5th to 6th decade of life. The most common site affected is the maxillary sinus followed by the nasal cavity [2]. Tumors of the sinonasal tract commonly present with symptoms that are identical to those caused by inflammatory sinus diseases, such as nasal obstruction, nasal discharge, epistaxis, headache, facial pain, and cheek swelling. ACC exhibits extensive local tissue infiltration and perineural spread, which results in a high rate of recurrence despite aggressive surgical resection. Classically, ACC has a distinct natural history of slow and indolent growth with late development of distant metastasis but tends to have local recurrence [3]. Those tumors are typically diagnosed late, and their proximity to vital structures (e.g., dura, brain, orbit, and central nerves) makes adequate oncological resection less likely [4]. Due to the high propensity of local invasion to adjacent vital anatomical structures (i.e., cranial nerves) and late diagnosis, ACC is associated with poor prognosis, intracranial extent, and positive surgical margins [5]. The mainstay of treatment of ACC is surgery, adjuvant radiation therapy is reserved in case of positive margins or advanced stage.
Adenoid cystic carcinomas, formerly known as cylindromas, are malignant epithelial tumors that arise from the exocrine glands, essentially the main and accessory salivary. The location in the nasal cavity is exceptional. The histological characteristics of this tumor make it difficult to manage.

\section{Case Report}

A 62-year-old female patient with underlying hypertension and diabetes initially presented for 3 years a permanent right unilateral nasal obstruction, associated with episodes of epistaxis and to clear anterior and posterior rhinorhées. There were no allergic rhinitis symptoms and smell disorders. There was no facial asymmetry, nor cheek numbness, loosening of teeth, blurring of vision, or limited mouth opening. There were also no headache, throat, or ear symptoms. Generally, the patient was stable.

There was blunted right nasolabial fold with vague mass overlying it. All cranial nerves examinations were unremarkable.

Nasoendoscope examination revealed a polypoid mass occupying the right nostril, friable, and bleed easily. The mass seems to be arising from the lateral nasal wall. The left nostril showed normal inferior and middle turbinate with clear osteomeatal 
complex and both Fossa of Rosen-Mueller were normal.

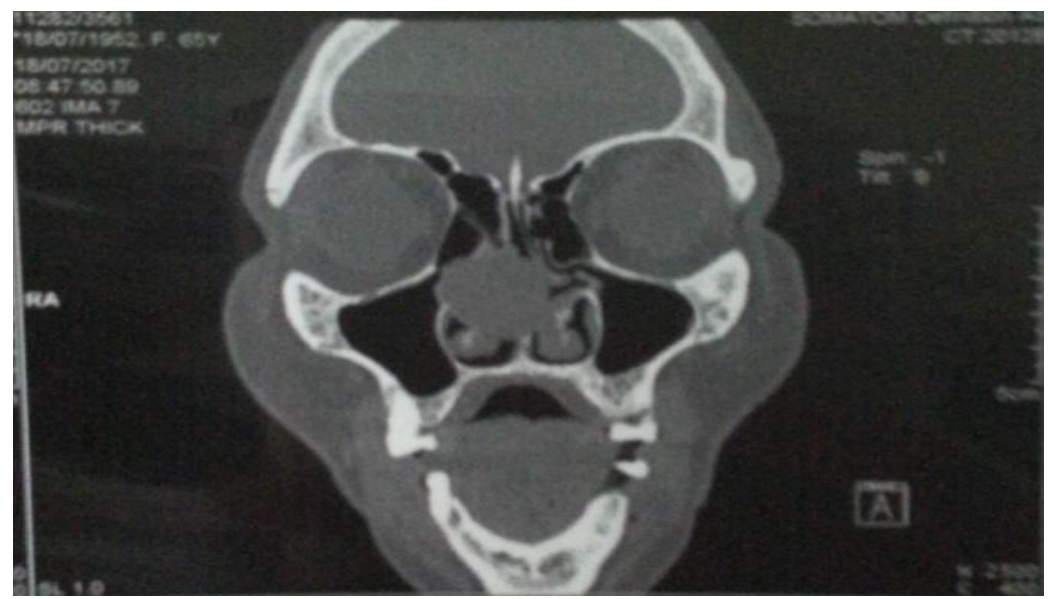

Fig-1: Coronary sinusal scan showing the process at right nasal cavity

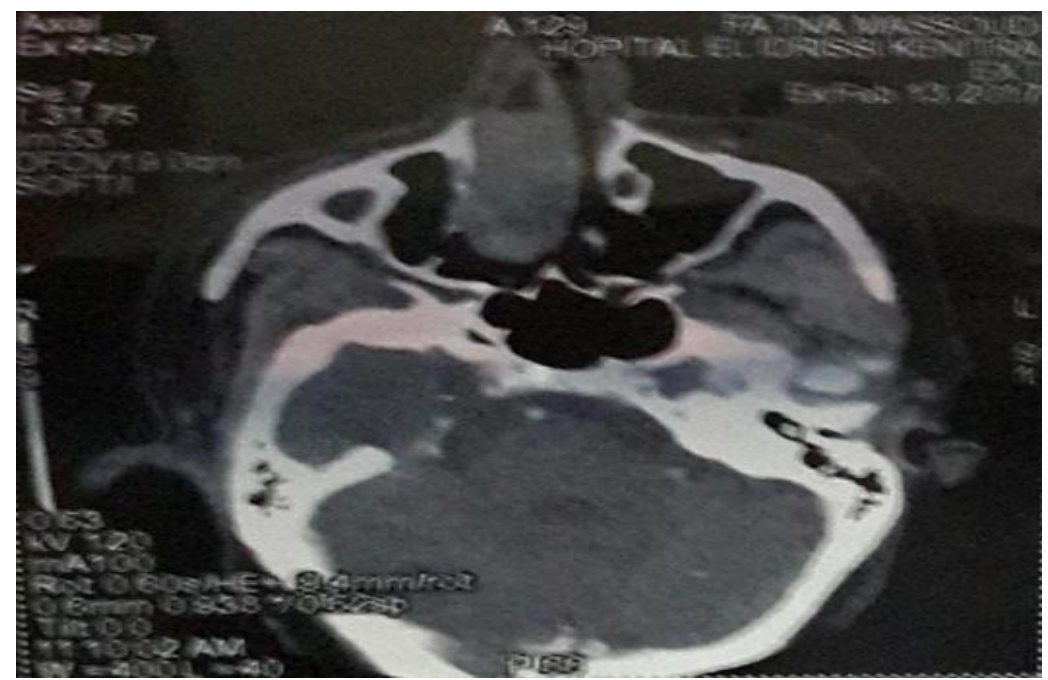

Fig-2: Sinusal axial scanner cutting showing the process of right nasal cavity

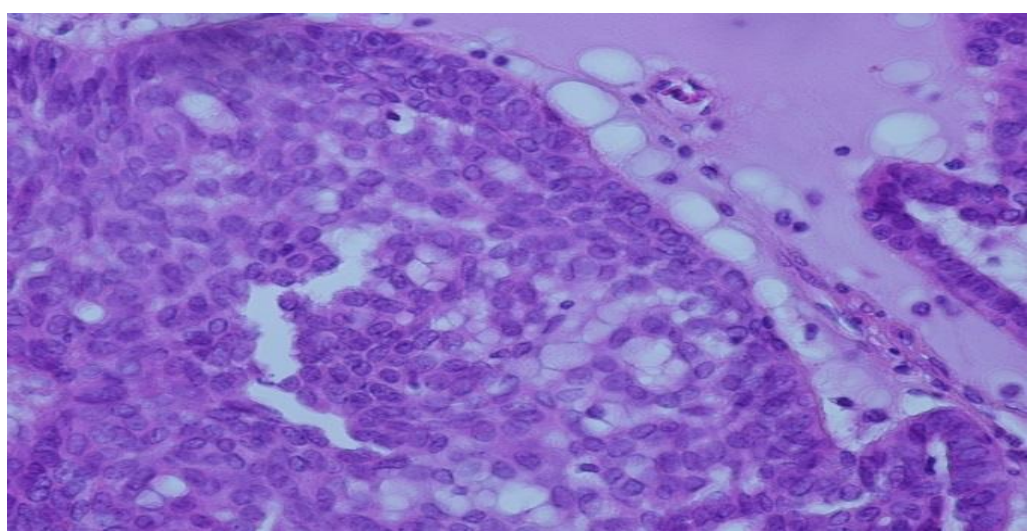

Fig-3: Anapath blade image showing malignant cells arranged in cribriform, tubular, cords, and solid pattern

The CT scan showed a mass of homogenous soft-tissue attenuation within the right nasal cavity at the expense of the middle turbinate with regular outline invading the nasal septum and extending into the left nasal fossa as well as the anterior right ethmoidal cells with the impression of sinonasal polyposis and differential of inverted papilloma or carcinoma. CT scan staging showed no sign of locoregional and distant metastases (figure 1.2).

The patient then underwent endoscopic resection of the tumor, near-total inferior turbinectomy, and middle meatal antrostomy under general anesthesia. Intraoperative findings showed a mass occupying the right nasal cavity, its pedicle originating from middle 
turbinate which easily bleeds. The anterior ethmoid cells were taken while the posterior ethmoid cells seemed normal.

Histopathological examination post-operative showed the tumor tissue fragment consists of malignant cells arranged in cribriform, tubular, cords, and solid pattern.

The patient underwent 35 fractions of radiotherapy for total60 $\mathrm{Gy}$, involving the mid-facial region and the nasosinusal cavities. During follow up 3 months post-radiotherapy, there was no local and regional recurrence.

\section{DISCUSSION}

Adenoid cystic carcinoma (CAK) is one of the most common malignant tumors of the salivary glands. However, its nasal location remains exceptional. It represents 0.5 to $1 \%$ of cancers in the otorhinolaryngological sphère $[6,7]$. They often interest patients between 40 and 60 years with an average of 51 years, a minimal preponderance of women is noted $[6,7]$. Furthermore, most patients at presentation are already at advanced stages of the disease [8]. The earlier the diagnosis and treatment received patients will have better outcomes in terms of morbidity, risk of residual, or recurrence of the disease. A few important factors that need to be considered in the treatment of sinonasal malignancy are oncological clearance of surgery, functional status of the patient.

Clinical manifestation of adenocystic carcinoma varies but the patient mainly presents with epistaxis and nasal blockage. As in our cases, its clinical presentation is classical. Sinonasal malignancy is commonly presented with nasal obstruction, followed by epistaxis and local spread symptoms such as auditory, nerve, and visual symptoms [9].

CAK is considered to be an infiltrating tumor with three possible profiles: glandular or cribriform, tubular, and solid. Solid type tumors should be distinguished from basal cell adenocarcinomas. Perineural or perivascular invasion is very characteristic. All adenoid cystic carcinomas are biologically aggressive and may give metastasis even years after surgical removal of the primary tumor. Lymph node metastases represent 3, 8, and 15\% depending on the series [6]. Perineural or perivascular invasion, mitotic activity, and cellular pleomorphism do not appear to have an exact correlation with the tumor prognosis. However, solid tumors seem to have a poorer prognosis, glandular, or tubular type tumors have a better prognosis.

The scan can guide the diagnosis, guide surgical resection, and define the target volume of radiotherapy.
Surgery is the mainstay of the treatment for sinonasal ACC. It can be either open, endoscopic, or a combined approach. The current paradigm of treatment of sinonasal malignancy is towards multimodality and multidisciplinary approaches. Usually, adjuvant chemoradiation is given 4-6 weeks after surgery. Our patient presented to us at stage T2NOM0 and underwent endoscopic approach tumor resection. The histological result showed an oncologic free tumor margin.

Although the study showed perineural and perivascular invasion is high in adenoid cystic carcinoma [10], in our case there was no invasion seen from imaging and histology.

Adenoid cystic carcinoma is associated with a good prognosis in stage I-II of the tumor when combined with multimodality treatment. Few factors give a favorable prognosis of ACC such as histopathological findings of solid type of tumor, absent of perineural or perivascular invasion, absence of distant metastases, site of the tumor as well as patient and treatment factors such as age and multi-modality treatment [11]. In our case upon follow up after radiotherapy, there was no local and distant tumor recurrence.

\section{CONCLUSiON}

Adenoid cystic carcinomas of the nasosinusal cavities are aggressive tumors associated with a high incidence of local recurrence and distant metastases regardless of the therapeutic modalities used. The radio surgical association offers the best chance of disease control.

\section{REFERENCES}

1. Ellington, C. L., Goodman, M., Kono, S. A., Grist, W., Wadsworth, T., Chen, A. Y., ... \& Beitler, J. J. (2012). Adenoid cystic carcinoma of the head and neck: incidence and survival trends based on 1973- 2007 Surveillance, Epidemiology, and End Results data. Cancer, 118(18), 4444-4451.

2. Gandhi, A. K., Roy, S., Biswas, A., Bhasker, S., Sharma, A., Thakar, A., \& Mohanti, B. K. (2015). Adenoid cystic carcinoma of head and neck: A single institutional analysis of 66 patients treated with multi-modality approach. Indian journal of medical and paediatric oncology: official journal of Indian Society of Medical \& Paediatric Oncology, 36(3), 166.

3. Sung, M. W., Kim, K. H., Kim, J. W., Min, Y. G., Seong, W. J., Roh, J. L., ... \& Park, S. W. (2003). Clinicopathologic predictors and impact of distant metastasis from adenoid cystic carcinoma of the head and neck. Archives of Otolaryngology-Head \& Neck Surgery, 129(11), 1193-1197. 
4. Patel, S. G., Singh, B., Polluri, A., Bridger, P. G., Cantu, G., Cheesman, A. D., ... \& Janecka, I. (2003). Craniofacial surgery for malignant skull base tumors: report of an international collaborative study. Cancer: Interdisciplinary International Journal of the American Cancer Society, 98(6), 1179-1187.

5. Mendenhall, W. M., Morris, C. G., Amdur, R. J., Werning, J. W., Hinerman, R. W., \& Villaret, D. B. (2004). Radiotherapy alone or combined with surgery for adenoid cystic carcinoma of the head and neck. Head \& Neck: Journal for the Sciences and Specialties of the Head and Neck, 26(2), 154162.

6. Liu, X. W., Xie, C. M., Li, H., Zhang, R., Geng, Z. J., Mo, Y. X., ... \& Wu, P. H. (2012). Nasopharyngeal adenoid cystic carcinoma: magnetic resonance imaging features in ten cases. Chinese journal of cancer, 31(1), 19.

7. Barry, B. (2000). La Lettre d'Oto-rhinolaryngologie et de chirurgie cervicofaciale. Données récentes en pathologie infectieuse ORL sur le traitement de première intention des infections ORL courantes. no.
8. Poursadegh, M., Poursadegh, F., Esmaeili, M., \& Bakhshaee, M. (2015). Epidemiological survey of sinonasal malignancy in North-East Iran. Iranian journal of otorhinolaryngology, 27(80), 225.

9. Thompson, L. D., Penner, C., Ho, N. J., Foss, R. D., Miettinen, M., Wieneke, J. A., ... \& Stelow, E. B. (2014). Sinonasal tract and nasopharyngeal adenoid cystic carcinoma: a clinicopathologic and immunophenotypic study of 86 cases. Head and neck pathology, 8(1), 88-109.

10. Hanna E, Vural E, Prokopakis E, Carrau R, Snyderman C, Weissman J. The sensitivity and specificity of high-resolution imaging in evaluating perineural spread of adenoid cystic carcinoma to the skull base. Archives of Otolaryngology-Head \& Neck Surgery. 2007 Jun 1;133(6):541-5.

11. Ko, Y. H., Lee, M. A., Hong, Y. S., Lee, K. S., Jung, C. K., Kim, Y. S., ... \& Kang, J. H. (2007). Prognostic factors affecting the clinical outcome of adenoid cystic carcinoma of the head and neck. Japanese journal of clinical oncology, 37(11), 805-811. 